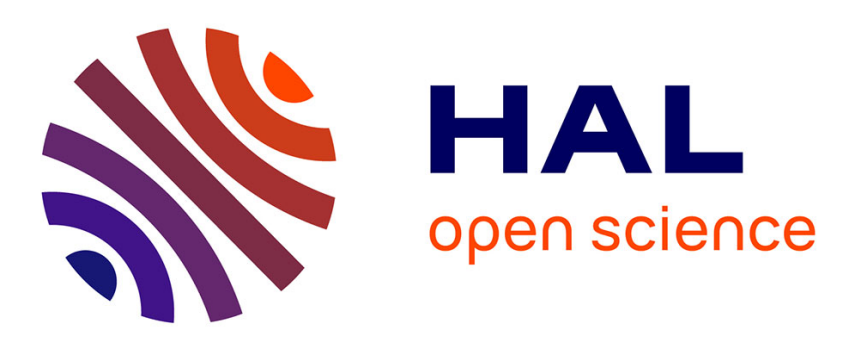

\title{
Multi-Sensorial Interface for 3D Teleoperation at Micro and Nanoscale
}

Nicolas Venant, Antoine Niguès, Florence Marchi, Michal Hrouzek, Fabio

Comin, Joël Chevrier, Jean-Loup Florens

\section{To cite this version:}

Nicolas Venant, Antoine Niguès, Florence Marchi, Michal Hrouzek, Fabio Comin, et al.. MultiSensorial Interface for 3D Teleoperation at Micro and Nanoscale. Haptics : generating and perceiving tangible sensations, Apr 2010, Amsterdam, Netherlands. pp.35-42. hal-00910683

\section{HAL Id: hal-00910683 \\ https://hal.science/hal-00910683}

Submitted on 3 Jul 2014

HAL is a multi-disciplinary open access archive for the deposit and dissemination of scientific research documents, whether they are published or not. The documents may come from teaching and research institutions in France or abroad, or from public or private research centers.
L'archive ouverte pluridisciplinaire HAL, est destinée au dépôt et à la diffusion de documents scientifiques de niveau recherche, publiés ou non, émanant des établissements d'enseignement et de recherche français ou étrangers, des laboratoires publics ou privés. 


\title{
Multi-Sensorial Interface for 3D Teleoperations at Micro and Nanoscale
}

\author{
Nicolas Venant ${ }^{1,3}$, Antoine Niguès ${ }^{2,3}$, Florence Marchi ${ }^{2,3}$, Michal Hrouzek ${ }^{4}$, \\ Fabio Comin ${ }^{3}$, Joël Chevrier ${ }^{2,3}$, and Jean-Loup Florens ${ }^{1}$ \\ 1 ICA, INPG, 46 av. Félix Viallet, 38031 Grenoble Cedex, France \\ ${ }^{2}$ Institut Néel, CNRS/Université Joseph Fourier, \\ 25 avenue des Martyrs, BP 166, 38042 Grenoble cedex 9, France \\ 3 ESRF, 6 rue Jules Horowitz, BP 220, 38043 Grenoble cedex 9, France \\ 4 Small Infinity, 6 rue Jules Horowitz, BP 220, 38043 Grenoble cedex 9, France \\ Jean-Loup.Florens@imag.fr
}

\begin{abstract}
This paper presents the design of a new tool for 3D manipulations at micro and nanoscale based on the coupling between a high performance haptic system (the ERGOS system) and two Atomic Force Microscope (AFM) probes mounted on quartz tuning fork resonators, acting as a nano tweezers. This unique combination provides new characteristics and possibilities for the localization and manipulation of (sub)micronic objects in 3 dimensions. The nano robot is controlled through a dual sensorial interface including 3D haptic and visual rendering, it is capable of performing a number of real-time tasks on different samples in order to analyse their dynamic effects when interacting with the AFM tips. The goal is then to be able to compare mechanical properties of different matters (stiffness of soft or hard matter) and to handle submicronic objects in 3 dimensions.
\end{abstract}

Key words: Atomic Force Microscope (AFM), ERGOS Force-Feedback transducer, Haptic Interface, Nanomanipulator, Real-Time, Tuning-Fork

\section{Introduction}

Consistent improvements of nanotechnologies have opened the way to the possibility of manipulating, modifying or measuring single nano-objects with very high efficiency. However, those actions still represent an important challenge [1, 2], often linked to the different set of rules that govern the nano world at variance with macro world (adhesion forces larger than gravity, for example) [3,4]. In such unusual world "easy" actions can turn in very difficult tasks, like drinking a glass of water in a weightlessness spaceship.

This paper deals with the development of a 3D multi-sensorial interface nanomanipulator using the ERGOS haptic system (bandwidth over $10 \mathrm{kHz}$ for a maximum force of $50 \mathrm{~N})$. This high performance haptic device is coupled with two nanometric tips glued on resonant quartz tuning forks as nanoforce sensors/ actuators. This unique and innovating combination between a haptic device and a 
dynamic force sensor provides new opportunities for nanomanipulation in comparison to a classical AFM cantilever. Used in static mode, conventional AFMs used in nanotweezers mix mechanically the two functions of action and force detection. To the contrary, the tuning fork can distinguish these two functions thanks to its high spring constant and the very small oscillations necessary for the detection (see Sect. 3).

Thanks to our custom-made interface, teleoperations in 1D, 2D and 3D are available via the coupling of real measurements with basic virtual information.

\section{Description of the Multisensorial Haptic Interface}

For teleoperation action at the micro and nanoscale, the user controls the position of one or two AFM probes (see Fig. 1) through a multisensorial interface. Indeed, the experimentalist could choose to perform action with a single nanofinger or two nano-fingers. To perform the micro-nano task, two complementary interfaces are available: haptic rendering (described in detail below) and visual rendering based on an optical objective mounted on a CCD-camera.

The haptic system is an ERGOS interface composed of two 3 degrees of freedom

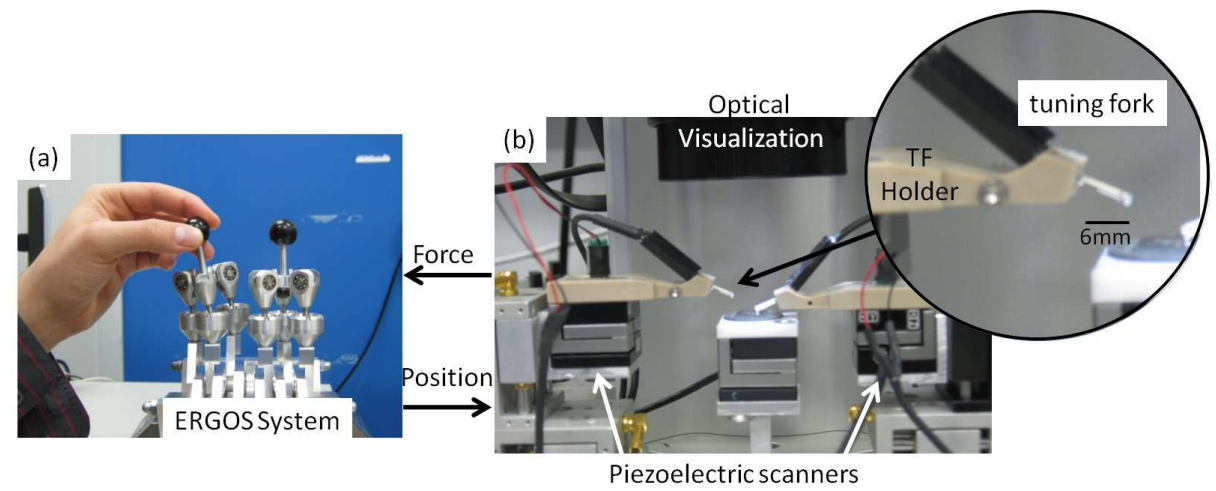

Fig. 1. (a) User space: 3D Joysticks from ERGOS system; (b) Task space: tuning fork AFM probes mounted on 3D piezoelectric scanners (MiniTritor from PiezoJena System)

Joysticks allowing each 3 translational motions in XYZ reference [6]. The full workspace of the slave robot (the 3D piezoelectric scanner) is a cube of $40 \mu \mathrm{m}$ side. Thus, a similar workspace geometry has been selected for the haptic system: a cube of $2 \mathrm{~cm}$ side for each joystick is mapped into the working space of the 3D piezoelectric scanner via a tuneable scaling factor that can vary the micro-cube side from less than $1 \mu \mathrm{m}$ to $40 \mu \mathrm{m}$. To create this cubic workspace, we imposed virtual boundaries by applying elastic forces to the joystick when it reaches a point of the cube surface in order to make the user feeling a wall. The stiffness 
of those elastic forces must be high enough to clearly define the inner working space. Given the variety of tasks and samples, this virtual environment must be flexible. So, the virtual environment parameters can be modified at will via the dashboard screens of the real-time software (programmed in Labview language). As an example the user can choose a rectangular, a flat or an arbitrary 1D direction as workspace or just freeze the joystick in one point. This virtual environment provides the basic virtual guides to assist the user in his/her action tasks.

\section{Quartz Tuning-Fork as a Force Sensor/Actuator}

Quartz Tuning Fork (TF) resonators replaces silicon cantilever in those atomic force specific applications where optics free detection is required [7]. Indeed, the main advantages of the TF are: (1) a compact ergonomics, quite compatible with other set-up (SEM, Scanning Electron Microscope, for example), (2) a high spring constant $K$ (around $45 \mathrm{kN} / \mathrm{m}$ versus few $\mathrm{N} / \mathrm{m}$ for standard AFM cantilever) that eliminates the position hysteresis of the tip when it gets in contact with the surface (the snap on and off is avoided). From a haptic point of view this high spring constant is a key issue to avoid the limitation due to the actuator stiffness in the force measurement and its haptic rendering with an AFM cantilever.

The use of a TF can allow to work in liquid environments [8], necessary for applications in biology.

The TF is used in dynamical mode: it is excited with sinusoidal signals close to the resonance frequency $\left(\omega_{0} \approx 32 k H z\right)$, and it behaves as an harmonic oscillator. When an interaction occurs between the tip and the surface, the resonance frequency is modified. The frequency shift is measured with a Phase Locked Loop (PLL). Considering just small oscillations, the frequency shift $\Delta f$ is related to the force gradient as [9]:

$$
\Delta f=\frac{\omega_{0}}{2 K} \frac{\partial F}{\partial z}
$$

where $\frac{\partial F}{\partial z}$ is the gradient of the tip-surface force along the $\mathrm{Z}$ direction.

The relationship between the force gradient and the $\mathrm{z}$ component $F_{z}$ of the force between the tip and the sample can be approximated by the equation [10]:

$$
F_{z}=A \frac{\partial F}{\partial z}
$$

where $A$ is the oscillations amplitude. The amplitude $A$ depends on the intensity of the TF exciting signal. In practice, a PI controller maintains $A$ constant (by adjusting the intensity of the exciting signal) leading to a proportional relationship between the force and the frequency shift:

$$
\Delta f=\frac{\omega_{0}}{2 K A} F_{z}
$$


Nevertheless, those approximations hold as long as the oscillation amplitude $A$ is small compared to the average tip-surface distance (one order of magnitude lower). This condition fits with typical experimental conditions where $A$ is about few angstroms while the average tip-surface distance is about few nanometers. The force feed-back is then calculate via a linear equation:

$$
F_{\text {feedback }}=C \cdot \Delta f+D
$$

where $C$ and $D$ are tuneable parameters adjusted to the sensitivity required by the experimentalist and the stability of the system (see Sect. 4).

This force is a $1 \mathrm{D}$ force and should naturally be applied in the $\mathrm{Z}$ direction to correspond to the TF measurement. We performed our $1 \mathrm{D}$ experiments in this configuration.

However, for 2D manipulations, a different configuration has been chosen. The $F_{\text {feedback }}$ is applied on the three directions of the user space. The direction of the force is imposed to be the opposite to the motion but does not correspond to real $3 \mathrm{D}$ force measurement in the task space as the TF can only measure force in 1D. In this condition, the user detects the contact whatever the direction he is moving on but he cannot distinguish the direction of the interaction force between the tip and the sample. Thanks to this configuration, a 3D haptic recognition of the sample has been achieved (see Figure 4, Section 5).

To overcome the lack of a complete information about the interaction, we plan to develop a virtual nanoscene associated to the real one. The virtual nanoscene will allow to simulate the associated force fields, and can be used in parallel with the teleoperation measurement in a Augmented Reality configuration. Via this configuration, the lateral force can be eventually evaluated. A similar approach have been already implement by others groups [11].

A second approach relies on the possibility of a dual excitation of the TF arms: the first in the vertical and the second in the lateral direction. From the resulting double information on the tip-sample interaction, a $3 \mathrm{D}$ reconstruction of the force field can be computed based on the approach implemented by Onal and Sitti [12] for a contact mode configuration with an AFM cantilever.

\section{Real Time Control}

The system setup supports two independent calculation systems as illustrated in Fig. 2. They are connected by an analogue signal (Frequency Shift).

The first system is a PLL from the commercial Nanonis electronics that is composed of a National Instruments FPGA card running at 5MHz. It controls the tuning-fork oscillations and measures the frequency shift (see Sect. 3). We notice here that the entire Nanonis Electronic System can also be configured to restitute classical AFM images.

The second element is a DSP card from Sheldon Instruments. This card links the master and slave robots, it has been programmed in LabView via QuView librairies supplied by Sheldon. The card is equipped with Input-Output channels of $16 \mathrm{bits}$ resolution at a sample rate of $10 \mathrm{kHz}$. To run in Real-Time configuration 


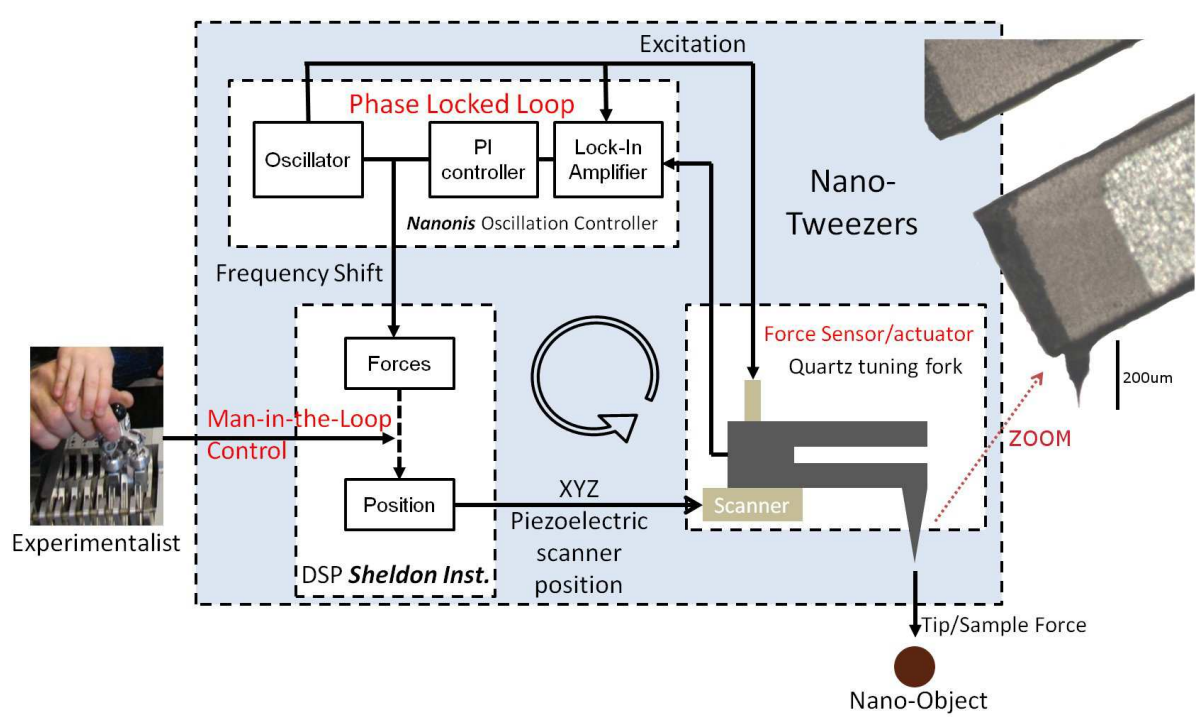

Fig. 2. Real-Time Control Loop: Oscillation Controller from Nanonis System and a DSP card from Sheldon Instruments to implement the man-in-the-loop control.

the algorithm also runs at $10 \mathrm{kHz}$ (single point mode).

Our system presents the ideal properties for the transparency of the set actuator/haptic interface: a high stiffness transducer, a high speed Real-Time control loop and a high quality features haptic device (ERGOS system, mechanical bandwidth over $10 \mathrm{kHz}$ ). This configuration assures that the user controls the tip through a rigid stick, thus making the reproduction of fast nano-phenomenons and hard-contact rendering possible.

The drawback is the increase of instabilities of the haptic interface. The problems of stability in haptic macro and nano-manipulation should not be underestimated due to the sensibility and time delays of the force sensor and the huge dynamics of the interaction stiffness between the tip and the sample. In the present work, the easiest solution has been implemented: we use a linear transformation to reproduced the nano interaction force on the haptic device (see Equ. 4). The amplification gain ( $C$ in Equ. 4$)$ is manually adjustable and has to be chosen carefully in order to avoid any instability. This parameter is sample and task dependant. The $D$ parameter in Equ. 4 is used to balance the static error (natural drift) of the $\Delta f$.

Better solutions to preserve the passivity of the system are under examination.

\section{Experimental Results}

Teleoperation experiments have been carried out in air at room temperature to validate the contact detection in $1 \mathrm{D}$ or $3 \mathrm{D}$ using well characterised samples. 


\section{D Basic teleoperation mode: grabbing of a microsphere}

A glass microsphere of $20 \mu m$ (diameter) has been successfully pushed, grabbed and held above the surface with the two tip system via visual rendering and joystick control (Fig. 3) but without force feedback. At the end, the user can maintain the micro-objet above the surface for several minutes.

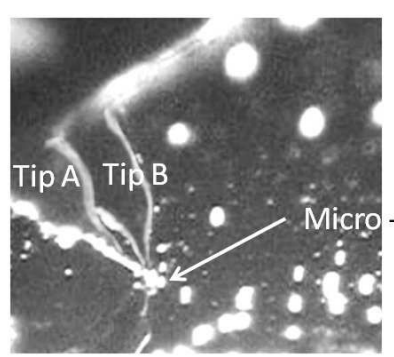

(a)

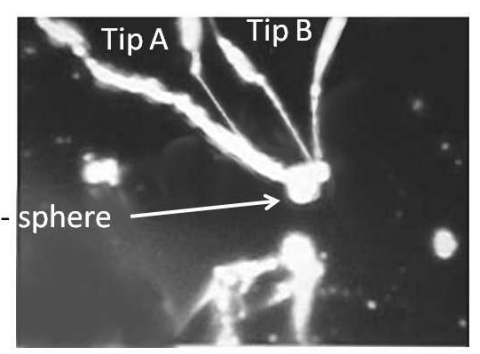

(b)

Fig. 3. Manipulation of a $20 \mu m$ diameter glass sphere: during the manipulation, the sphere has been pushed; (a) grabbed; (b) held over the surface.

\section{D Basic teleoperation mode: manipulation of a microsphere}

A latex sphere of $2 \mu \mathrm{m}$ of diameter has been moved by one tip, using a 3D force feedback in the user space. This 3D force, built on the user's motion (see Section 3) enables to explore haptically the sample. After the sphere has been haptically localized (Fig. 4(a)), it has been moved on the surface for few microns without damage (Fig. 4(b)).

Despite the easiness to manipulate the sphere with 3D force feedback, a strong limitation still persists: the micro-object is manipulated by a $3 \mathrm{D}$ displacement of the tip, but the complete picture of the interaction is still unknown as only the $\mathrm{Z}$ component of the force is measured.

\section{D teleoperation: Exploration of the sample elasticity}

The interaction force between a tip and a PDMS circular membrane (soft sample) has been probed and the corresponding force on the haptic device recorded (Fig.5). Thanks to the high spring constant of the TF, the frequency shift variation could be attributed only to the sample deformation. This experiment cannot be realised with an AFM cantilever due to the limited cantilever stiffness. 

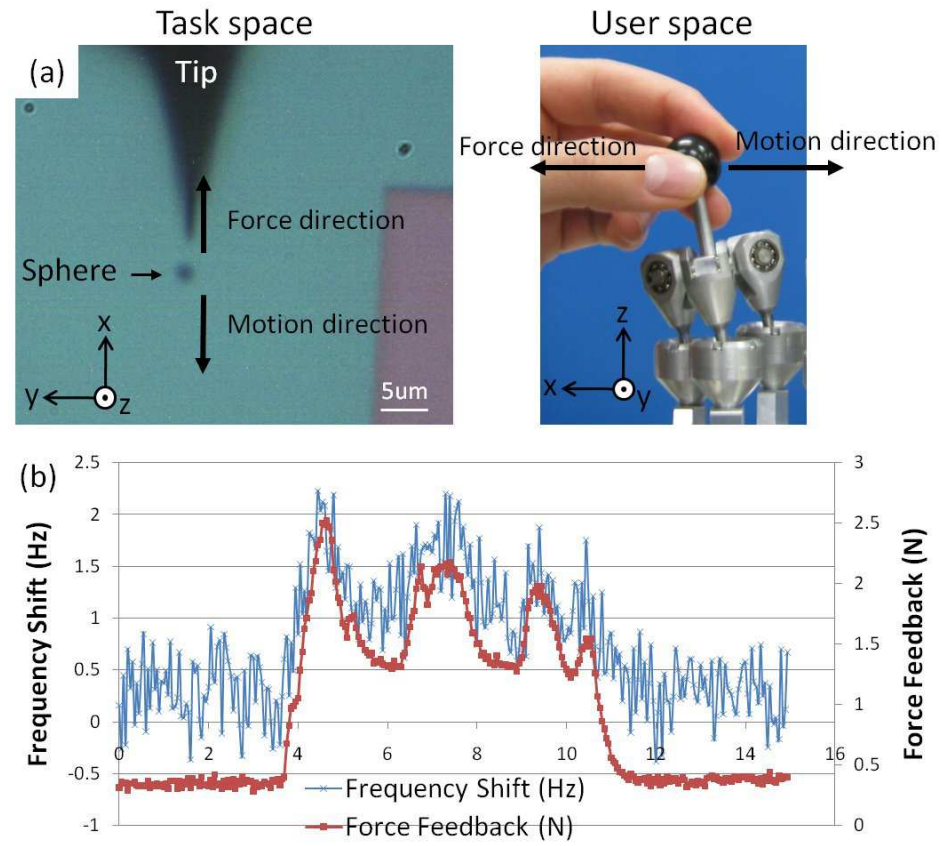

Fig. 4. Manipulation of a $2 \mu m$ latex sphere: (a) the sphere has been haptically "felt" via a $3 \mathrm{D}$ force feeback and then pushed for few microns along the surface; (b) Frequency Shift and Force Feedback signals in function of time (s) during the displacement of the sphere.

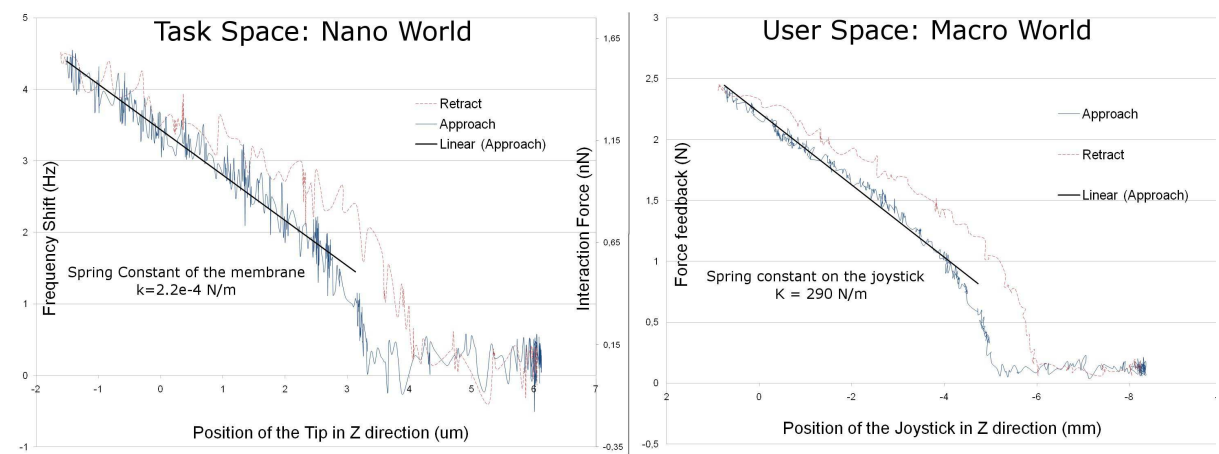

Fig. 5. Approach-Retract Curve over a PDMS (polydimethylsiloxane) circular membrane with the haptic interface manipulator. Membrane diameter and thickness: $5 \mathrm{~mm}$; $10 \mu \mathrm{m}$. 


\section{Conclusion}

The set of experiments validates the efficiency of the dual independent probes as a gripping micro/nano-prehensor and its coupling with a Real-Time haptic interface. All the experiments have been accomplished several times with good repeatability and no need of particular dexterity. In addition, this work demonstrated the relevance of frequency shift measurements in the task space to transfer them in force information in the user space. This result paves the way for the nanomanipulation in dynamic mode with rigid micro-transducer.

In the next step, a more complex simulated nano environment will be implemented to provide additional virtual information like realistic 3D force field and visual surface rendering.

Acknowledgments. Curie Institut, Paris, for providing PDMS samples.

\section{References}

1. Aruk, B., Hashimoto, H., Sitti, M.: Man-Machine Interface for Micro-Nano Manipulation with an AFM Probe. IEEE-NANO 2001, 151-156 (2001)

2. Xie, H., Haliyo, D.S., Regnier, S.: A versatile atomic force microscope for threedimentional nanomanipulation and nanoassembly. Nanotechnology 2009, (2009)

3. Marliere, S., Urma, D., Florens, J.L., Marchi, F.: Multi-sensorial interaction with a nano-scale phenomenon: the force curve. Proceedings of EuroHaptics 2004, Munich Germany, June 5-7, 246-252 (2004)

4. Saeidpourazar, R., Jalili, N.: Towards fused vision and force robust feedback control of nanorobotic-based manipulation and grasping. Mechatronics, vol 18, 566-577 (2008)

5. Sitti, M.: Survey of Nanomanipulation Systems. IEEE-NANO 2001, 75-80 (2001)

6. Florens, J.L., Lucianni, A., Cadoz, C., Castagne, N.: ERGOS: Multi-degrees of Freedom and Versatile Force-Feedback Panoply. Proceedings of EuroHaptics 2004, Munich Germany, June 5-7, 356-360 (2004)

7. Scheler, T., Rodrigues, M., Cornelius, T. W., Mocuta, C., Malachias, A., MagalhesPaniago, R., Comin, F., Chevrier, J. and Metzger, T. H.: APPLIED PHYSICS LETTERS 94, 023109 (2009)

8. Lee, L., Schaller, R., Haber, L.H., Saykally, R.J.: High Spatial Resolution Imaging with Near-Field Scanning Optical Microscopy in Liquids. Anal. Chem., 73 (21), pp 50155019 (2001)

9. Barbic, M., Eliason, L., Ranshaw, J.: Femto-Newton force sensitivity quartz tuning fork sensor. Sensors and actuators. A, Physical 136, 564-566, (2007)

10. Rensen, W.H.: Tuning Fork Tunes, Exploring new scanning probe techniques. pp $31(2002)$

11. Vogl, W., Ma, B.K.L., Sitti, M.: Augmented Reality User Interface for an Atomic Force Microscope-Based Nanorobotic System. IEEE Transactions on Nanotechnology, Vol. 5, 397-406 (2006)

12. Onal, C.D., Sitti, M.: Teleoperated 3-D Force Feedback From the Nanoscale With an Atomic Force Microscope. IEEE Transactions on Nanotechnology, Vol. 9, No. 1 (2010) 